\title{
Blow-Up of Solutions for a Coupled Nonlinear Viscoelastic Equation with Degenerate Damping Terms: Without Kirchhoff Term
}

\author{
Salah Mahmoud Boulaaras $\left(\mathbb{D},{ }^{1,2}\right.$ Abdelbaki Choucha, ${ }^{3}$ Mohamed Abdalla ${ }^{(\mathbb{D}},{ }^{4,5}$ \\ Karthikeyan Rajagopal $\mathbb{}^{6},{ }^{6}$ and Sahar Ahmed Idris ${ }^{7,8}$ \\ ${ }^{1}$ Department of Mathematics, College of Sciences and Arts, ArRas, Qassim University, Buraydah, Saudi Arabia \\ ${ }^{2}$ Laboratory of Fundamental and Applied Mathematics of Oran (LMFAO), University of Oran 1, Oran 31000, Algeria \\ ${ }^{3}$ Department of Mathematics, Faculty of Exact Sciences, University of El Oued, El Oued, Algeria \\ ${ }^{4}$ Department of Mathematics, Faculty of Science, King Khalid University, Abha 61471, Saudi Arabia \\ ${ }^{5}$ Mathematics Department, Faculty of Science, South Valley University, Qena 83523, Egypt \\ ${ }^{6}$ Center for Nonlinear Systems, Chennai Institute of Technology, Chennai, Tamil Nadu 600069, India \\ ${ }^{7}$ College of Industrial Engineering, King Khalid University, Abha 62529, Saudi Arabia \\ ${ }^{8}$ Department of Mathematics, College of Sciences, Juba University, Juba, Sudan
}

Correspondence should be addressed to Salah Mahmoud Boulaaras; s.boularas@qu.edu.sa

Received 9 August 2021; Accepted 3 November 2021; Published 22 November 2021

Academic Editor: Sundarapandian Vaidyanathan

Copyright (C) 2021 Salah Mahmoud Boulaaras et al. This is an open access article distributed under the Creative Commons Attribution License, which permits unrestricted use, distribution, and reproduction in any medium, provided the original work is properly cited.

In this work, we consider a quasilinear system of viscoelastic equations with degenerate damping and source terms without the Kirchhoff term. Under suitable hypothesis, we study the blow-up of solutions.

\section{Introduction}

In this paper, we consider the following problem:

$$
\left\{\begin{array}{l}
\left|u_{t}\right|^{\eta} u_{t t}-\Delta u+\int_{0}^{t} h_{1}(t-s) \Delta u(s) d s+\left(|u|^{k}+|v|^{l}\right)\left|u_{t}\right|^{j-1} u_{t} \\
=f_{1}(u, v),(x, t) \in \Omega \times(0, T), \\
\left.\left|v_{t}\right|^{\eta} v_{t t}-\Delta v+\int_{0}^{t} h_{2}(t-s) \Delta v(s) d s+\left(|v|^{\theta}+|u|\right\rangle\right)\left|v_{t}\right|^{s-1} v_{t} \\
=f_{2}(u, v),(x, t) \in \Omega \times(0, T), \\
u(x, t)=v(x, t)=0, \quad(x, t) \in \partial \Omega \times(0, T), \\
u(x, 0)=u_{0}(x), u_{t}(x, 0)=u_{1}(x), \quad x \in \Omega, \\
v(x, 0)=v_{0}(x), v_{t}(x, 0)=v_{1}(x), \quad x \in \Omega,
\end{array}\right.
$$

where $k, l, \theta, \varrho \geq 0 ; j, s \geq 1$ for $N=1,2$, and $0 \leq j, s \leq(N+$ $2 / N-2)$ for $N \geq 3$; and $\eta \geq 0$ for $N=1,2$, and $0<\eta \leq(2 / N-2)$ for $N \geq 3$; and $h_{i}():. R^{+} \longrightarrow R^{+}$ $(i=1,2)$ are positive relaxation functions which will be specified later. $\left(|(.)|^{a}+|(.)|^{b}\right)\left|(.)_{t}\right|^{\tau-1}(.)_{t}$ is the degenerate damping term, and

$$
\left\{\begin{array}{l}
f_{1}(u, v)=a_{1}|u+v|^{2(p+1)}(u+v)+b_{1}|u|^{p} \cdot u \cdot|v|^{p+2}, \\
f_{2}(u, v)=a_{1}|u+v|^{2(p+1)}(u+v)+b_{1}|v|^{p} \cdot v \cdot|u|^{p+2} .
\end{array}\right.
$$

The motivation of our problem firstly is by the initial boundary value problem for the quasilinear equation of the form

$$
\left|u_{t}\right|^{\eta} u_{t t}-\Delta u+\int_{0}^{t} h(t-s) \Delta u(s) d s+g\left(u, u_{t}\right)=f(u) .
$$


This type of problem is frequently found in some mathematical models in applied sciences, especially in the theory of viscoelasticity. Problem (3) has been studied by various authors, and several results concerning asymptotic behavior and blow-up have been studied (case $\eta \geq 0$ ). For example, in the case $\left(g\left(u, u_{t}\right)=0\right)$, problem (3) has been investigated in [1] and the author proved the blow-up result. In the case $\left(g\left(u, u_{t}\right)=0\right)$ of boundary value problem and in the presence of the dispersion term $\left(-\Delta u_{t t}\right)$, Liu [2] studied a general decay of solutions. And, in [3], the authors applied the potential well method to indicate the global existence and uniform decay of solutions $\left(g\left(u ; u_{t}\right)\right)=0$ instead of $\left.\Delta u_{t}\right)$. Furthermore, the authors obtained a blow-up result. In the case $\left(g\left(u, u_{t}\right)=\left|u_{t}\right|^{m} u_{t}\right)$, in [4], Wu studied a general decay of solution. Later, the same author in [5] considered the same problem but $\left(g\left(u, u_{t}\right)=u_{t}\right)$ and discussed the decay rate of solution. Recently, in [6], the authors proved the existence of global solution and a general stability result.

There are several works in case $(\eta=0)$, where the authors have studied the blow-up of solutions of problem (3) (for example, see [3, 7-12]).

For a coupled system, He [13] considered the following problem:

$$
\left\{\begin{array}{l}
\left|u_{t}\right|^{\eta} u_{t t}-\Delta u+\int_{0}^{t} h_{1}(t-s) \Delta u(s) d s-\Delta u_{t t}+g_{1}\left(u, u_{t}\right) \\
\quad=f_{1}(u, v), \\
\left|v_{t}\right|^{\eta} v_{t t}-\Delta v+\int_{0}^{t} h_{2}(t-s) \Delta v(s) d s-\Delta v_{t t}+g_{2}\left(v, v_{t}\right) \\
\quad=f_{2}(u, v),
\end{array}\right.
$$

where $\quad \eta>0 ; j, s \geq 2 ; \quad$ and $\quad g_{1}\left(u, u_{t}\right)=\left|u_{t}\right|^{j-2} u_{t}$ and $g_{2}\left(v, v_{t}\right)=\left|v_{t}\right|^{s-2} v_{t}$. The author proved general and optimal decay of solutions. Then, in [14], the author investigated the same problem without damping term and established a general decay of solutions. Furthermore, the author obtained a blow-up of solutions. In addition, in problem (1) with $\eta=0$, in [15], Wu proved a general decay of solutions. Later, in [16], Piskin and Ekinci established a general decay and blow-up of solutions with nonpositive initial energy for problem (1) case (Kirchhoff type). In recent years, some other authors investigate the hyperbolic type system with degenerate damping terms (see [17-20]). Very recently, in the presence of the dispersion term $\left(-\Delta u_{t t}\right)$, our problem (1) has been studied in [21]. Under some restrictions on the initial datum and standard conditions on relaxation functions, the authors have established the global existence and proved the general decay of solutions.

Based on all of the abovementioned discussion, we believe that the combination of these terms of damping (memory term, degenerate damping, and source terms) constitutes a new problem worthy of study and research, different from the above that we will try to shed light on, especially the blow-up of solutions.

Our paper is divided into several sections: In Section 2, we lay down the hypotheses, concepts, and lemmas we need.
In Section 3, we prove our main result. Finally, we give some concluding remarks in the last section.

\section{Preliminaries}

We prove the blow-up result under the following suitable assumptions:

(A1) $h_{i}: \mathbb{R}_{+} \longrightarrow \mathbb{R}_{+}$are differentiable and decreasing functions such that

$$
h_{i}(t) \geq 0,1-\int_{0}^{\infty} h_{i}(s) d s=l_{i}>0, \quad i=1,2 .
$$

(A2) There exist a constants $\xi_{1}, \xi_{2}>0$ such that

$$
h_{i}^{\prime}(t) \leq-\xi_{i} h_{i}(t), \quad t \geq 0, i=1,2 .
$$

Lemma 1. There exists a function $F(u, v)$ such that

$$
\begin{aligned}
& F(u, v)=\frac{1}{2(p+2)}\left[u f_{1}(u, v)+v f_{2}(u, v)\right] \\
& \frac{1}{2(p+2)}\left[a_{1}|u+v|^{2(p+2)}+2 b_{1}|u v|^{p+2}\right] \geq 0,
\end{aligned}
$$

where

$$
\begin{aligned}
& \frac{\partial F}{\partial u}=f_{1}(u, v), \\
& \frac{\partial F}{\partial v}=f_{2}(u, v) .
\end{aligned}
$$

We take $a_{1}=b_{1}=1$ for convenience.

Lemma 2 (see [18]). There exist two positive constants $c_{0}$ and $c_{1}$ such that

$$
\begin{aligned}
& \frac{c_{0}}{2(p+2)}\left(|u|^{2(p+2)}+|v|^{2(p+2)}\right) \leq F(u, v) \\
& \leq \frac{c_{1}}{2(p+2)}\left(|u|^{2(p+2)}+|v|^{2(p+2)}\right) .
\end{aligned}
$$

Now, we state the local existence theorem that can be established by combining arguments of $[13,16]$.

Theorem 1. Assume (5) and (6) hold. Let

$$
\left\{\begin{array}{l}
-1<p<\frac{4-n}{n-2}, \quad n \geq 3 \\
p \geq-1, \quad n=1,2 .
\end{array}\right.
$$

Then, for any initial datum,

$$
\left(u_{0}, u_{1}, v_{0}, v_{1}\right) \in \mathscr{H} .
$$

Problem (1) has a unique solution, for some $T>0$ : 


$$
\begin{gathered}
u, v \in C\left([0, T] ; H^{2}(\Omega) \cap H_{0}^{1}(\Omega)\right), \\
u_{t} \in C\left([0, T] ; H_{0}^{1}(\Omega)\right) \cap L^{j+1}(\Omega), \\
v_{t} \in C\left([0, T] ; H_{0}^{1}(\Omega)\right) \cap L^{s+1}(\Omega),
\end{gathered}
$$

where

$$
\mathscr{H}=H_{0}^{1}(\Omega) \times L^{2}(\Omega) \times H_{0}^{1}(\Omega) \times L^{2}(\Omega) .
$$

Now, we define the energy functional.

Lemma 3. Assume (5), (6), and (10) hold; let $(u, v)$ be a solution of (1); then, $E(t)$ is nonincreasing, that is,

$$
\begin{aligned}
E(t)= & \frac{1}{\eta+2}\left[\left\|u_{t}\right\|_{\eta+2}^{\eta+2}+\left\|v_{t}\right\|_{\eta+2}^{\eta+2}\right], \\
& +\frac{1}{2}\left[\left(1-\int_{0}^{t} h_{1}(s) d s\right)\|\nabla u\|_{2}^{2}\right. \\
& \left.+\left(1-\int_{0}^{t} h_{2}(s) d s\right)\|\nabla u\|_{2}^{2}\right], \\
& +\frac{1}{2}\left[\left(h_{1} o \nabla u\right)(t)+\left(h_{2} o \nabla u\right)(t)\right]-\int_{\Omega} F(u, v) \mathrm{d} x,
\end{aligned}
$$

which satisfies

$$
\begin{aligned}
E^{\prime}(t) \leq & \frac{1}{2}\left[\left(h_{1}^{\prime} o \nabla u\right)(t)+\left(h_{2}^{\prime} o \nabla u\right)\right](t) \\
& -\frac{1}{2}\left[h_{1}(t)\|\nabla u\|_{2}^{2}+h_{2}(t)\|\nabla u\|_{2}^{2}\right], \\
& -\int_{\Omega}\left(|u|^{k}+|v|^{l}\right)\left|u_{t}\right|^{j+1} d x-\int_{\Omega}\left(|v|^{\theta}+|u|^{\varrho}\right)\left|v_{t}\right|^{s+1} d x,
\end{aligned}
$$

$\leq 0$.

Proof. By multiplying the first and second equations in (1) by $u_{t}, v_{t}$ and integrating over $\Omega$, we get

$$
\begin{aligned}
& \frac{d}{d t}\left\{\frac{1}{\eta+2}\left\|u_{t}\right\|_{\eta+2}^{\eta+2}+\frac{1}{\eta+2}\left\|v_{t}\right\|_{\eta+2}^{\eta+2}+\frac{1}{2}\left(-1 \int_{0}^{t} h_{1}(s) d s\right)\|\nabla u\|_{2}^{2}+\frac{1}{2}\left(-1 \int_{0}^{t} h_{2}(s) d s\right)\|\nabla u\|_{2}^{2}+\frac{1}{2}\left(h_{1} o \nabla u\right)(t)\right. \\
& \left.\quad+\frac{1}{2}\left(h_{2} o \nabla u\right)(t)-\int_{\Omega} F(u, v) d x\right\}, \\
& \quad=-\int_{\Omega}\left(|u|^{k}+|v|^{l}\right)\left|u_{t}\right|^{j+1} d x-\int_{\Omega}\left(|v|^{\theta}+|u|^{\varrho}\right)\left|v_{t}\right|^{s+1} d x+\frac{1}{2}\left(h_{1}^{\prime} o \nabla u\right)-\frac{1}{2} h_{1}(t)\|\nabla u\|_{2}^{2}+\frac{1}{2}\left(h_{2}^{\prime} o \nabla u\right)-\frac{1}{2} h_{2}(t)\|\nabla u\|_{2}^{2} .
\end{aligned}
$$

We obtain (14) and (15).

First, we define the functional as

\section{Blow-Up}

In this section, we prove the blow-up result of solution of problem (1).

$$
\begin{aligned}
\mathbb{Q}(t)= & -E(t)=-\frac{1}{\eta+2}\left[\left\|u_{t}\right\|_{\eta+2}^{\eta+2}+\left\|v_{t}\right\|_{\eta+2}^{\eta+2}\right]-\frac{1}{2}\left(1-\int_{0}^{t} h_{1}(s) d s\right)\|\nabla u\|_{2}^{2}+\frac{1}{2}\left(1-\int_{0}^{t} h_{2}(s) d s\right)\|\nabla v\|_{2}^{2} \\
& -\frac{1}{2}\left[\left(h_{1} o \nabla u\right)(t)+\left(h_{2} o \nabla u\right)(t)\right] \\
& +\frac{1}{2(p+2)}\left[\|u+v\|_{2(p+2)}^{2(p+2)}+\|u v\|_{2(p+2)}^{2(p+2)}\right] .
\end{aligned}
$$


Theorem 4. Assume that (5), (6), and (10) hold, and suppose that $E(0)<0$ and

$$
2(p+2)>\max \{k+j+1 ; l+j+1 ; \theta+s+1 ; \varrho+s+1\} .
$$

Then, the solution of problem (1) blows up in finite time.

Proof. From (14), we have

$$
E(t) \leq E(0) \leq 0 .
$$

Therefore,

$$
\begin{aligned}
\mathbb{H}^{\prime}(t)= & -E^{\prime}(t) \geq \int_{\Omega}\left(|u|^{k}+|v|^{l}\right)\left|u_{t}\right|^{j+1} d x \\
& +\int_{\Omega}\left(|v|^{\theta}+|u|^{\varrho}\right)\left|v_{t}\right|^{s+1} d x .
\end{aligned}
$$

Hence,

$$
\begin{aligned}
& \mathbb{H}^{\prime}(t) \geq \int_{\Omega}\left(|u|^{k}+|v|^{l}\right)\left|u_{t}\right|^{j+1} d x \geq 0, \\
& \mathbb{H}^{\prime}(t) \geq \int_{\Omega}\left(|v|^{\theta}+|u|^{\varrho}\right)\left|v_{t}\right|^{s+1} d x \geq 0 .
\end{aligned}
$$

By (9) and (17), we have

$$
\begin{aligned}
0 \leq \mathbb{H}(0) \leq \mathbb{W}(t) & \leq \frac{1}{2(p+2)}\left[\|u+v\|_{2(p+2)}^{2(p+2)}+2\|u v\|_{2(p+2)}^{2(p+2)}\right], \\
& \leq \frac{c_{1}}{2(p+2)}\left[\left\|u_{t}\right\|_{\eta+2}^{\eta+2}+\left\|v_{t}\right\|_{\eta+2}^{\eta+2}\right]
\end{aligned}
$$

We set

$$
\mathscr{K}(t)=\mathbb{H}^{1-\alpha}+\frac{\mathcal{E}}{\eta+1} \int_{\Omega}\left[u\left|u_{t}\right|^{\eta} u_{t}+v\left|v_{t}\right|^{\eta} v_{t}\right] d x,
$$

where $\varepsilon>0$ is to be assigned later and

$$
0<\alpha<\min \left\{\left(1-\frac{1}{2(p+2)}-\frac{1}{\eta+2}\right), \frac{2 p+3-(k+j)}{2 j(p+2)}, \frac{2 p+3-(l+j)}{2 j(p+2)}, \frac{2 p+3-(\theta+s)}{2 s(p+2)}, \frac{2 p+3-(\varrho+s)}{2 s(p+2)}\right\}<_{1}
$$

By multiplying the first and second equations in (1) by $u, v$ and with a derivative of (23), we get

$$
\begin{aligned}
& \mathscr{K}^{\prime}(t)=(1-\alpha) \mathbb{\boxplus}^{-\alpha} \mathbb{Z}^{\prime}(t)+\frac{\varepsilon}{\eta+1}\left(\left\|u_{t}\right\|_{\eta+2}^{\eta+2}+\left\|v_{t}\right\|_{\eta+2}^{\eta+2}\right)+\underbrace{\varepsilon \int_{\Omega} \nabla u \int_{0}^{t} g(t-s) \nabla u(s) d s d x}_{J_{1}}+\underbrace{\varepsilon \int_{\Omega} \nabla v \int_{0}^{t} h(t-s) \nabla u(s) d s d x}_{J_{2}} \\
& -\underbrace{\varepsilon \int_{\Omega}\left(|u|^{k}+|v|^{l}\right)\left|u_{t}\right|^{j-1} u_{t} \cdot u d x}_{J_{3}}-\underbrace{\varepsilon \int_{\Omega}\left(|v|^{\theta}+|u|^{\varrho}\right)\left|v_{t}\right|^{s-1} v_{t} \cdot v d x}_{J_{4}}-\varepsilon\left(\|\nabla u\|_{2}^{2}+\|\nabla v\|_{2}^{2}\right)+\underbrace{\varepsilon\left[\|u+v\|_{2(p+2)}^{2(p+2)}+2\|u v\|_{2(p+2)}^{2(p+2)}\right]}_{J_{5}},
\end{aligned}
$$

where we have

$$
\begin{aligned}
J_{1}= & \varepsilon \int_{0}^{t} h_{1}(t-s) d s \int_{\Omega} \nabla u \cdot(\nabla u(s)-\nabla u(t)) d x d s \\
& +\varepsilon \int_{0}^{t} h_{1}(s) d s\|\nabla u\|_{2}^{2}, \\
\geq & \frac{\varepsilon}{2} \int_{0}^{t} h_{1}(s) d s\|\nabla u\|_{2}^{2}-\frac{\varepsilon}{2}\left(h_{1} o \nabla u\right), \\
J_{2}= & \varepsilon \int_{0}^{t} h_{2}(t-s) d s \int_{\Omega} \nabla u \cdot(\nabla u(s)-\nabla u(t)) d x d s \\
& +\varepsilon \int_{0}^{t} h_{2}(s) d s\|\nabla u\|_{2}^{2}, \\
\geq & \frac{\varepsilon}{2} \int_{0}^{t} h_{2}(s) d s\|\nabla u\|_{2}^{2}-\frac{\varepsilon}{2}\left(h_{2} o \nabla u\right) .
\end{aligned}
$$

From (25), we find that

$$
\begin{aligned}
\mathscr{K}^{\prime}(t) \geq & (1-\alpha) \mathbb{H}^{-\alpha} \mathbb{W}^{\prime}(t)+\frac{\varepsilon}{\eta+1}\left(\left\|u_{t}\right\|_{\eta+2}^{\eta+2}+\left\|v_{t}\right\|_{\eta+2}^{\eta+2}\right) \\
& -\varepsilon\left[\left(1-\frac{1}{2} \int_{0}^{t} h_{1}(s) d s\right)\|\nabla u\|_{2}^{2}\right. \\
& \left.+\left(1-\frac{1}{2} \int_{0}^{t} h_{2}(s) d s\right)\|\nabla v\|_{2}^{2}\right] \\
& -\frac{\varepsilon}{2}\left(h_{1} o \nabla u\right)-\frac{\varepsilon}{2}\left(h_{2} o \nabla u\right)-J_{3}-J_{4}+J_{5} .
\end{aligned}
$$

At this point, we use Young's inequality; for $\delta>0$,

$$
X Y \leq \frac{\delta^{\alpha} X^{\alpha}}{\alpha}+\frac{\delta^{-\beta} X^{\beta}}{\beta}, \alpha, \beta>0, \frac{1}{\alpha}+\frac{1}{\beta}=1 \text {, }
$$

We get that for $\delta_{1}, \delta_{2}>0$, 


$$
\begin{gathered}
\left.\left.|u| u_{t}\right|^{j-1} u_{t}\left|\leq \frac{\delta_{1}^{j+1}}{j+1}\right| u_{t}\right|^{j+1}+\frac{j}{j+1} \delta_{1}^{-(j+1 / j)}\left|u_{t}\right|^{j+1}, \\
\left.\left.|v| v_{t}\right|^{s-1} v_{t}\left|\leq \frac{\delta_{2}^{s+1}}{s+1}\right| v_{t}\right|^{s+1}+\frac{s}{s+1} \delta_{2}^{-(s+1 / s)}\left|v_{t}\right|^{s+1}
\end{gathered}
$$

Hence, we have

$$
\begin{aligned}
J_{3} \leq & \varepsilon \frac{\delta_{1}^{j+1}}{j+1} \int_{\Omega}\left(|u|^{k}+|v|^{l}\right)\left|u_{t}\right|^{j+1} d x \\
& +\varepsilon \frac{j \delta_{1}^{-(j+1 / j)}}{j+1} \int_{\Omega}\left(|u|^{k}+|v|^{l}\right)\left|u_{t}\right|^{j+1} d x d x \\
J_{4} \leq & \varepsilon \frac{\delta_{2}^{s+1}}{s+1} \int_{\Omega}\left(|v|^{\theta}+|u|^{\varrho}\right)\left|v_{t}\right|^{s+1} d x \\
& +\varepsilon \frac{s \delta_{2}^{-(s+1 / s)}}{s+1} \int_{\Omega}\left(|v|^{\theta}+|u|^{\varrho}\right)\left|v_{t}\right|^{s+1} d x
\end{aligned}
$$

Therefore, using (21) and by setting $\delta_{1}, \delta_{2}$ so that

$$
\begin{aligned}
& \frac{j \delta_{1}^{-(j+1 / j)}}{j+1}=\frac{\kappa \llbracket^{-\alpha}(t)}{2}, \\
& \frac{s \delta_{2}^{-(s+1 / s)}}{s+1}=\frac{\kappa \coprod^{-\alpha}(t)}{2},
\end{aligned}
$$

and substituting in (28), we get

$$
\begin{aligned}
\mathscr{K}^{\prime}(t) \geq & {[(1-\alpha)-\varepsilon \kappa] \mathbb{\boxplus}^{-\alpha} \mathbb{\boxplus}^{\prime}(t)+\frac{\varepsilon}{\eta+1}\left(\left\|u_{t}\right\|_{\eta+2}^{\eta+2}+\left\|v_{t}\right\|_{\eta+2}^{\eta+2}\right)-\varepsilon\left[\left(1-\frac{1}{2} \int_{0}^{t} h_{1}(s) d s\right)\|\nabla u\|_{2}^{2}+\left(1-\frac{1}{2} \int_{0}^{t} h_{2}(s) d s\right)\|\nabla v\|_{2}^{2}\right] } \\
& -\frac{\varepsilon}{2}\left(h_{1} o \nabla u\right)-\frac{\varepsilon}{2}\left(h_{2} o \nabla u\right)-\varepsilon C_{1}(\kappa) \mathbb{\boxplus}^{\alpha j}(t) \int_{\Omega}\left(|u|^{k}+|v|^{l}\right)\left|u_{t}\right|^{j+1} d x-\varepsilon C_{2}(\kappa) \mathbb{\boxplus}^{\alpha j}(t) \int_{\Omega}\left(|v|^{\theta}+|u|^{\varrho}\right)\left|v_{t}\right|^{s+1}+J_{5},
\end{aligned}
$$

where

$$
\begin{aligned}
& C_{1}(\kappa):=\left(\frac{2 j}{\kappa(j+1)}\right)^{j+1} \frac{1}{j+1}, \\
& C_{2}(\kappa):=\left(\frac{2 s}{\kappa(s+1)}\right)^{s+1} \frac{1}{s+1} .
\end{aligned}
$$

We have

$$
\begin{aligned}
& \int_{\Omega}\left(|u|^{k}+|v|^{l}\right)\left|u_{t}\right|^{j+1} d x=\|u\|_{k+j+1}^{k+j+1}+\int_{\Omega}|v|^{l}|u|^{j+1} d x \\
& \int_{\Omega}\left(|v|^{\theta}+|u|^{\varrho}\right)\left|v_{t}\right|^{s+1} d x=\|v\|_{\theta+s+1}^{\theta+s+1}+\int_{\Omega}|u|^{\varrho}|v|^{s+1} d x
\end{aligned}
$$

By Young's inequality, we find that for $\delta_{3}, \delta_{4}>0$,

$$
\begin{aligned}
\int_{\Omega}|v|^{l}|u|^{j+1} d x \leq & \frac{l}{l+j+1} \delta_{3}^{(l+j+1 / l)}\|v\|_{l+j+1}^{l+j+1} \\
& +\frac{j+1}{l+j+1} \delta_{3}^{-(l+j+1 / l)}\|u\|_{l+j+1}^{l+j+1}, \\
\int_{\Omega}|u|^{\varrho}|v|^{s+1} d x \leq & \frac{\varrho}{\varrho+s+1} \delta_{4}^{(\varrho+s+1 / \varrho)}\|u\|_{\varrho+s+1}^{\varrho+s+1} \\
& +\frac{s+1}{\varrho+s+1} \delta_{4}^{-((\varrho+s+1 / \varrho))}\|v\|_{\varrho+s+1}^{\varrho+s+1} .
\end{aligned}
$$

Hence,

$$
\begin{aligned}
& \mathbb{M}^{\alpha j}(t) \int_{\Omega}\left(|u|^{k}+|v|^{l}\right)\left|u_{t}\right|^{j+1} d x \leq \mathbb{T}^{\alpha j}(t)\|u\|_{k+j+1}^{k+j+1}+\frac{l \mathbb{T}^{\alpha j}(t)}{l+j+1} \delta_{3}^{(l+j+1 / l)}\|v\|_{l+j+1}^{l+j+1}+\frac{(j+1) \mathbb{M}^{\alpha j}(t)}{l+j+1} \delta_{3}^{-((l+j+1 / l))}\|u\|_{l+j+1}^{l+j+1}, \\
& \mathbb{\mathbb { H } ^ { \alpha j }}(t) \int_{\Omega}\left(|v|^{\theta}+|u|^{\varrho}\right)\left|v_{t}\right|^{s+1} d x \leq \mathbb{T}^{\alpha s}(t)\|v\|_{\theta+s+1}^{\theta+s+1}+\frac{\varrho \mathbb{\boxplus}^{\alpha s}(t)}{\varrho+s+1} \delta_{4}^{(\varrho+s+1 / \varrho)}\|u\|_{\varrho+s+1}^{\varrho+s+1}+\frac{(s+1) \mathbb{W}^{\alpha s}(t)}{\varrho+s+1} \delta_{4}^{-((\varrho+s+1 / \varrho))}\|v\|_{\varrho+s+1}^{\varrho+s+1} .
\end{aligned}
$$

Since (10) holds, we obtain the following by using (22) and (24): 
$\mathbb{T}^{\alpha j}(t)\|u\|_{k+j+1}^{k+j+1} \leq c_{1}\left(\|u\|_{2(p+2)}^{2 \alpha j(p+2)+k+j+1}+\|v\|_{2(p+2)}^{2 \alpha j(p+2)}\|u\|_{k+j+1}^{k+j+1}\right)$, $\mathbb{\boxplus}^{\alpha j}(t)\|v\|_{k+j+1}^{k+j+1} \leq c_{2}\left(\|v\|_{2(p+2)}^{2 \alpha j(p+2)+k+j+1}+\|u\|_{2(p+2)}^{2 \alpha j(p+2)}\|v\|_{k+j+1}^{k+j+1}\right)$, $\mathbb{T}^{\alpha s}(t)\|v\|_{\theta+s+1}^{\theta+s+1} \leq c_{3}\left(\|v\|_{2(p+2)}^{2 \alpha s(p+2)+\theta+s+1}+\|u\|_{2(p+2)}^{2 \alpha s(p+2)}\|v\|_{\theta+s+1}^{\theta+s+1}\right)$, $\mathbb{T}^{\alpha s}(t)\|u\|_{\theta+s+1}^{\theta+s+1} \leq c_{4}\left(\|u\|_{2(p+2)}^{2 \alpha s(p+2)+\theta+s+1}+\|v\|_{2(p+2)}^{2 \alpha s(p+2)}\|u\|_{\theta+s+1}^{\theta+s+1}\right)$,

for some positive constants $c_{i}, i=1, . .4$. By using (24) and the algebraic inequality

$$
B^{\varsigma} \leq(B+1) \leq\left(1+\frac{1}{b}\right)(B+b), \forall B>0,0<\varsigma<1, b>0,
$$

we have, $\forall t>0$,

$$
\begin{aligned}
& \|u\|_{2(p+2)}^{2 \alpha j(p+2)+k+j+1} \leq d\left(\|u\|_{2(p+2)}^{2(p+2)}+\mathbb{U}(0)\right) \leq d\left(\|u\|_{2(p+2)}^{2(p+2)}+\mathbb{U}(t)\right), \\
& \|v\|_{2(p+2)}^{2 \alpha j(p+2)+k+j+1} \leq d\left(\|v\|_{2(p+2)}^{2(p+2)}+\mathbb{U}(0)\right) \leq d\left(\|v\|_{2(p+2)}^{2(p+2)}+\mathbb{U}(t)\right), \\
& \|v\|_{2(p+2)}^{2 \alpha s(p+2)+\theta+s+1} \leq d\left(\|v\|_{2(p+2)}^{2(p+2)}+\mathbb{U}(0)\right) \leq d\left(\|v\|_{2(p+2)}^{2(p+2)}+\mathbb{U}(t)\right), \\
& \|u\|_{2(p+2)}^{2 \alpha s(p+2)+\theta+s+1} \leq d\left(\|u\|_{2(p+2)}^{2(p+2)}+\mathbb{U}(0)\right) \leq d\left(\|u\|_{2(p+2)}^{2(p+2)}+\mathbb{U}(t)\right),
\end{aligned}
$$

where $d=1+(1 / \mathbb{M}(0))$. Also, since

$$
(X+Y)^{\gamma} \leq C\left(X^{\gamma}+Y^{\gamma}\right), X, Y>0, \gamma>0,
$$

we conclude that

$$
\begin{aligned}
& \|v\|_{2(p+2)}^{2 \alpha j(p+2)}\|u\|_{k+j+1}^{k+j+1} \leq c_{5}\left(\|v\|_{2(p+2)}^{2(p+2)}+\|u\|_{2(p+2)}^{2(p+2)}\right), \\
& \|u\|_{2(p+2)}^{2 \alpha j(p+2)}\|v\|_{k+j+1}^{k+j+1} \leq c_{6}\left(\|u\|_{2(p+2)}^{2(p+2)}+\|v\|_{2(p+2)}^{2(p+2)}\right), \\
& \|u\|_{2(p+2)}^{2 \alpha s(p+2)}\|v\|_{\theta+s+1}^{\theta+s+1} \leq c_{7}\left(\|v\|_{2(p+2)}^{2(p+2)}+\|u\|_{2(p+2)}^{2(p+2)}\right), \\
& \|v\|_{2(p+2)}^{2 \alpha s(p+2)}\|u\|_{\theta+s+1}^{\theta+s+1} \leq c_{8}\left(\|v\|_{2(p+2)}^{2(p+2)}+\|u\|_{2(p+2)}^{2(p+2)}\right) .
\end{aligned}
$$

Substituting (40) and (42) in (38), we get

$$
\begin{aligned}
& \mathbb{T}^{\alpha j}(t)\|u\|_{k+j+1}^{k+j+1} \leq c_{9}\left(\|v\|_{2(p+2)}^{2(p+2)}\right)+\left(\|u\|_{2(p+2)}^{2(p+2)}+\mathbb{U}(t)\right), \\
& \mathbb{T}^{\alpha j}(t)\|v\|_{k+j+1}^{k+j+1} \leq c_{10}\left(\|v\|_{2(p+2)}^{2(p+2)}\right)+\left(\|u\|_{2(p+2)}^{2(p+2)}+\mathbb{U}(t)\right), \\
& \mathbb{T}^{\alpha j}(t)\|v\|_{k+j+1}^{k+j+1} \leq c_{11}\left(\|v\|_{2(p+2)}^{2(p+2)}\right)+\left(\|u\|_{2(p+2)}^{2(p+2)}+\mathbb{U}(t)\right), \\
& \mathbb{T}^{\alpha j}(t)\|u\|_{k+j+1}^{k+j+1} \leq c_{12}\left(\|v\|_{2(p+2)}^{2(p+2)}\right)+\left(\|u\|_{2(p+2)}^{2(p+2)}+\mathbb{U}(t)\right) .
\end{aligned}
$$

Hence, by fixing $\delta_{3}, \delta_{4}>0$, we get

$$
\begin{aligned}
& \mathbb{T}^{\alpha j}(t) \int_{\Omega}\left(|u|^{k}+|v|^{l}\right)\left|u_{t}\right|^{j+1} d x \leq M_{1}\left(1+\frac{l \delta_{3}^{(l+j+1 / l)}}{l+j+1}+\frac{(j+1) \delta_{3}^{-(l+j+1 / l)}}{l+j+1}\right)\left(\|v\|_{2(p+2)}^{2(p+2)}+\|u\|_{2(p+2)}^{2(p+2)}+\mathbb{U}(t)\right), \\
& \mathbb{T}^{\alpha s}(t) \int_{\Omega}\left(|v|^{\theta}+|u|^{\varrho}\right)\left|v_{t}\right|^{s+1} d x \leq M_{2}\left(1+\frac{\varrho \delta_{4}^{(\varrho+s+1 / \varrho)}}{\varrho+s+1}+\frac{(s+1) \delta_{4}^{-(\varrho+s+1 / \varrho)}}{\varrho+s+1}\right)\left(\|v\|_{2(p+2)}^{2(p+2)}+\|u\|_{2(p+2)}^{2(p+2)}+\mathbb{U}(t)\right),
\end{aligned}
$$

for some constants $M_{1}, M_{2}>0$.

Now, for $0<a<1$, from (17),

$$
\begin{aligned}
J_{5}= & \varepsilon\left[\|u+v\|_{2(p+2)}^{2(p+2)}+2\|u v\|_{p+2}^{p+2}\right]=\varepsilon a\left[\|u+v\|_{2(p+2)}^{2(p+2)}+2\|u v\|_{p+2}^{p+2}\right]+\frac{2 \varepsilon(p+2)(1-a)}{\eta+2}\left(\left\|u_{t}\right\|_{\eta+2}^{\eta+2}+\left\|v_{t}\right\|_{\eta+2}^{\eta+2}\right) \\
& +\varepsilon(p+2)(1-a)\left(1-\int_{0}^{t} g(s) d s\right)\|\nabla u\|_{2}^{2}+\varepsilon(p+2)(1-a)\left(1-\int_{0}^{t} h(s) d s\right)\|\nabla v\|_{2}^{2} \\
& -\varepsilon(p+2)(1-a)\left(\left(h_{1} o \nabla u\right)+\left(h_{2} o \nabla u\right)\right)+\varepsilon 2(p+2)(1-a) \mathbb{U}(t) .
\end{aligned}
$$

Substituting in (33) and by using (9), we get 


$$
\begin{aligned}
\mathscr{K}^{\prime}(t) \geq & \{(1-a)-\varepsilon \kappa\} \mathbb{\boxplus}^{-\alpha} \mathbb{\boxplus}^{\prime}(t)+\varepsilon\left\{\frac{2 \varepsilon(p+2)(1-a)}{\eta+2}+\frac{1}{\eta+1}\right\}\left(\left\|u_{t}\right\|_{\eta+2}^{\eta+2}+\left\|v_{t}\right\|_{\eta+2}^{\eta+2}\right), \\
& +\varepsilon\left\{(p+2)(1-a)\left(1-\int_{0}^{t} h_{1}(s) d s\right)-\left(1-\frac{1}{2} \int_{0}^{t} h_{1}(s) d s\right)\right\}\|\nabla u\|_{2}^{2} \\
& +\varepsilon\left\{(p+2)(1-a)\left(1-\int_{0}^{t} h_{2}(s) d s\right)-\left(1-\frac{1}{2} \int_{0}^{t} h_{2}(s) d s\right)\right\}\|\nabla v\|_{2}^{2} \\
& +\varepsilon\left\{(p+2)(1-a)-\frac{1}{2}\right\}\left(h_{1} o \nabla u+h_{2} o \nabla v\right)+\varepsilon\left\{c_{0} a-\left(M_{3} C_{1}(\kappa)+M_{4} C_{2}(\kappa)\right)\right\}\left(\|u\|_{2(p+2)}^{2(p+2)}+\|v\|_{2(p+2)}^{2(p+2)}\right) \\
& +\varepsilon\left\{2(p+2)(1-a)-\left(M_{3} C_{1}(\kappa)+M_{4} C_{2}(\kappa)\right)\right\} \mathbb{W}(t),
\end{aligned}
$$

where

$$
\begin{aligned}
& M_{3}:=M_{1}\left(1+\frac{l \delta_{3}^{(l+j+1 / l)}}{l+j+1}+\frac{(j+1) \delta_{3}^{-(l+j+1 / l)}}{l+j+1}\right)>0, \\
& M_{4}:=M_{2}\left(1+\frac{\varrho \delta_{4}^{(\varrho+s+1 / \varrho)}}{\varrho+s+1}+\frac{(s+1) \delta_{4}^{-(\varrho+s+1 / \varrho)}}{\varrho+s+1}\right)>0 .
\end{aligned}
$$

In this stage, we take $a>0$ small enough so that

$$
\lambda_{1}=(p+2)(1-a)-1>0,
$$

and we assume that

$$
\begin{aligned}
\max \left\{\int_{0}^{\infty} h_{1}(s) d s, \int_{0}^{\infty} h_{2}(s) d s\right\} & <\frac{(p+2)(1-a)-1}{((p+2)(1-a)-(1 / 2))} \\
& =\frac{2 \lambda_{1}}{2 \lambda_{1}+1}
\end{aligned}
$$

gives

$$
\begin{aligned}
& \lambda_{2}=\left\{((p+2)(1-a)-1)-\int_{0}^{t} h_{1}(s) d s\left((p+2)(1-a)-\frac{1}{2}\right)\right\}>0, \\
& \left.\lambda_{3}=\left\{((p+2)(1-a)-1)-\int_{0}^{t} h_{2}(s) d s((p+2)(1-a))-\frac{1}{2}\right)\right\}>0 .
\end{aligned}
$$

Then, we choose $\kappa$ so large such that

$$
\begin{aligned}
& \lambda_{4}=a c_{0}-\left(M_{3} C_{1}(\kappa)+M_{4} C_{2}(\kappa)\right)>0, \\
& \lambda_{5}=2(p+2)(1-a)-\left(M_{3} C_{1}(\kappa)+M_{4} C_{2}(\kappa)\right)>0 .
\end{aligned}
$$

Finally, we fix $\kappa$ and $a$ and we appoint $\varepsilon$ small enough so that

$$
\lambda_{6}=(1-\alpha)-\varepsilon \kappa>0 .
$$

Thus, for some $\beta>0$, estimate (46) becomes

$$
\mathscr{K}^{\prime}(t) \geq \beta\left\{\mathbb{W}(t)+\left\|u_{t}\right\|_{\eta+2}^{\eta+2}+\left\|v_{t}\right\|_{\eta+2}^{\eta+2}+\|\nabla u\|_{2}^{2}+\|\nabla v\|_{2}^{2}+\left(h_{1} o \nabla u\right)+\left(h_{2} o \nabla v\right)+\|u\|_{2(p+2)}^{2(p+2)}+\|u\|_{2(p+2)}^{2(p+2)}\right\} .
$$

By (9), for some $\beta_{1}>0$, we obtain

$$
\mathscr{K}^{\prime}(t) \geq \beta_{1}\left\{\mathbb{U}(t)+\left\|u_{t}\right\|_{\eta+2}^{\eta+2}+\left\|v_{t}\right\|_{\eta+2}^{\eta+2}+\|\nabla u\|_{2}^{2}+\|\nabla v\|_{2}^{2}+\left(h_{1} o \nabla u\right)+\left(h_{2} o \nabla v\right)+\|u+v\|_{2(p+2)}^{2(p+2)}+2\|u v\|_{(p+2)}^{(p+2)}\right\}
$$

and

$$
\begin{aligned}
& \left|\int_{\Omega}\left(u\left|u_{t}\right|^{\eta} u_{t}+v\left|v_{t}\right|^{\eta} v_{t}\right) d x\right|^{(1 / 1-\alpha)} \\
& \quad \leq C\left[\|u\|_{2(p+2)}^{(\theta / 1-\alpha)}+\left\|u_{t}\right\|_{\eta+2}^{(\mu / 1-\alpha)}+\|v\|_{2(p+2)}^{(\theta / 1-\alpha)}+\left\|v_{t}\right\|_{\eta+2}^{(\mu / 1-\alpha)}\right],
\end{aligned}
$$$$
\mathscr{K}(t) \geq \mathscr{K}(0)>0, t>0 .
$$

Next, using Hölder's and Young's inequalities, we have 
where $(1 / \mu)+(1 / \theta)=1$.

We take $\mu=(\eta+2)(1-\alpha)$, to get

$$
\frac{\theta}{1-\alpha}=\frac{\eta+2}{(1-\alpha)(\eta+2)-1} \leq 2(p+2) \text {. }
$$

Subsequently, by using (24), (22), and (39), we obtain

$$
\begin{array}{r}
\|u\|_{2(p+2)}^{(\eta+2 /(1-\alpha)(\eta+2)-1)} \leq d\left(\|u\|_{2(p+2)}^{2(p+2)}+\mathbb{H}(t)\right), \\
\|v\|_{2(p+2)}^{(\eta+2 /(1-\alpha)(\eta+2)-1)} \leq d\left(\|v\|_{2(p+2)}^{2(p+2)}+\mathbb{H}(t)\right), \forall t \geq 0 .
\end{array}
$$

Therefore,

$$
\begin{aligned}
& \left|\int_{\Omega}\left(u\left|u_{t}\right|^{\eta} u_{t}+v\left|v_{t}\right|^{\eta} v_{t}\right) d x\right|^{(1 / 1-\alpha)} \\
& \quad \leq c_{13}\left[\|u\|_{2(p+2)}^{2(p+2)}+\|v\|_{2(p+2)}^{2(p+2)}+\left\|u_{t}\right\|_{\eta+2}^{\eta+2}+\left\|v_{t}\right\|_{\eta+2}^{\eta+2}+H(t)\right] .
\end{aligned}
$$

Hence, by substituting (59) into (23), we get

$$
\begin{aligned}
\mathscr{K}^{(1 / 1-\alpha)}(t) & =\left(\mathbb{T}^{1-\alpha}+\frac{\varepsilon}{\eta+1} \int_{\Omega}\left(u\left|u_{t}\right|^{\eta} u_{t}+v\left|v_{t}\right|^{\eta} v_{t}\right) d x\right)^{(1 / 1-\alpha)}, \\
& \leq c\left(\mathbb{H}(t)+\left|\int_{\Omega}\left(u\left|u_{t}\right|^{\eta} u_{t}+v\left|v_{t}\right|^{\eta} v_{t}\right) d x\right|^{(1 / 1-\alpha)}\right), \\
& \leq c\left(\mathbb{T}(t)+\left\|u_{t}\right\|_{\eta+2}^{\eta+2}+\left\|v_{t}\right\|_{\eta+2}^{\eta+2}+\|u\|_{2(p+2)}^{2(p+2)}+\|v\|_{2(p+2)}^{2(p+2)}\right), \\
& \leq c\left(\mathbb{T}(t)+\left\|u_{t}\right\|_{\eta+2}^{\eta+2}+\left\|v_{t}\right\|_{\eta+2}^{\eta+2}+\|\nabla u\|_{2}^{2}+\|\nabla v\|_{2}^{2}+\left(h_{1} o \nabla u\right)+\left(h_{2} o \nabla v\right)+\|u\|_{2(p+2)}^{2(p+2)}+\|v\|_{2(p+2)}^{2(p+2)}\right) .
\end{aligned}
$$

From (53) and (60), we get

$$
\mathscr{K}^{\prime}(t) \geq \lambda \mathscr{K}^{(1 / 1-\alpha)}(t)
$$

where $\lambda>0$, and this depends only on $\beta$ and $c$.

By integration of (61), we obtain

$$
\mathscr{K}^{(\alpha / 1-\alpha)}(t) \geq \frac{1}{\mathscr{K}^{(-\alpha / 1-\alpha)}(0)-\lambda(\alpha / 1-\alpha) t} .
$$

Hence, $\mathscr{K}(t)$ blows up in time

$$
T \leq T^{*}=\frac{1-\alpha}{\lambda \alpha \mathscr{K}^{(\alpha / 1-\alpha)}(0)} .
$$

Then, the proof is completed.

\section{Conclusion}

The objective of this work is the study of the blow-up of solutions for a quasilinear viscoelastic system with degenerate damping. This type of problem is frequently found in some mathematical models in applied sciences, especially in the theory of viscoelasticity. What interests us in this current work is the combination of these terms of damping (memory term, degenerate damping, and source terms), which dictates the emergence of these terms in the system.

In the next work, we will try using the same method with the same problem in addition to other damping terms (dispersion term, Balakrishnan-Taylor damping, and delay term).

\section{Data Availability}

No data were used.

\section{Conflicts of Interest}

The authors declare that they have no conflicts of interest.

\section{Acknowledgments}

Sahar Ahmed Idris extends appreciation to the Deanship of Scientific Research at King Khalid University for funding this work through research groups program under grant RGP2/53/42.

\section{References}

[1] H. Song, "Global nonexistence of positive initial energy solutions for a viscoelastic wave equation," Nonlinear Analysis, vol. 125, pp. 260-269, 2015.

[2] W. Liu, "General decay and blow-up of solution for a quasilinear viscoelastic problem with nonlinear source," Nonlinear Analysis: Theory, Methods \& Applications, vol. 73, no. 6, pp. 1890-1904, 2010.

[3] G. Liang, Y. Zhaoqin, and L. Guonguang, "Blow up and global existence for a nonlinear viscoelastic wave equation with strong damping and nonlinear damping and source terms," Applied Mathematics, vol. 6, pp. 806-816, 2015.

[4] S. T. Wu, "General decay of solutions for a viscoelastic equation with nonlinear damping and source terms," Acta Mathematica Scientia, vol. 318, pp. 1436-1448, 2011. 
[5] S. T. Wu, "General decay of energy for a viscoelastic equation with damping and source terms," Taiwanese Journal of Mathematics, vol. 16, no. 1, pp. 113-128, 2012.

[6] H. Yang, S. Fang, F. Liang, and M. Li, "A general stability result for second order stochastic quasilinear evolution equations with memory," Boundary Value Problems, vol. 62, pp. 1-16, 2020.

[7] A. M. Alghamdi, S. Gala, M. A. Ragusa, and Z. Zhang, "A regularity criterion for the $3 \mathrm{D}$ density-dependent $\mathrm{MHD}$ equations," Bulletin of the Brazilian Mathematical Society, New Series, vol. 52, no. 2, pp. 241-251, 2020.

[8] A. Mohammad Alghamdi, S. Gala, S. Gala, C. Qian, and M. Alessandra Ragusa, "The anisotropic integrability logarithmic regularity criterion for the 3D MHD equations," Electronic research archive, vol. 28, no. 1, pp. 183-193, 2020.

[9] A. Choucha, D. Ouchenane, and S. Boulaaras, "Blow-up of a nonlinear viscoelastic wave equation with distributed delay combined with strong damping and source terms," Journal of Nonlinear Functional Analysis, vol. 31, 2020.

[10] H. Song and D. Xue, "Blow up in a nonlinear viscoelastic wave equation with strong damping," Nonlinear Analysis: Theory, Methods \& Applications, vol. 109, pp. 245-251, 2014.

[11] H. Song and C. Zhong, "Blow-up of solutions of a nonlinear viscoelastic wave equation," Nonlinear Analysis: Real World Applications, vol. 11, no. 5, pp. 3877-3883, 2010.

[12] A. Zarai, A. Draifia, and S. Boulaaras, "Blow-up of solutions for a system of nonlocal singular viscoelastic equations," Applicable Analysis, vol. 97, no. 13, pp. 2231-2245, 2018.

[13] L. He, "On decay and blow-up of solutions for a system of equations," Applicable Analysis, vol. 100, no. 11, pp. 2449-2477, 2019.

[14] L. He, "On decay of solutions for a system of coupled viscoelastic equations," Acta Applicandae Mathematica, vol. 167, no. 1, pp. 171-198, 2020.

[15] S.-T. Wu, "General decay of solutions for a nonlinear system of viscoelastic wave equations with degenerate damping and source terms," Journal of Mathematical Analysis and Applications, vol. 406, no. 1, pp. 34-48, 2013.

[16] E. Pişkin and F. Ekinci, "General decay and blow up of solutions for coupled viscoelastic equation of Kirchhoff type with degenerate damping terms," Mathematical Methods in the Applied Sciences, vol. 42, no. 16, pp. 5468-5488, 2019.

[17] M. M. Cavalcanti, V. N. Domingos Cavalcanti, and J. Ferreira, "Existence and uniform decay for a non-linear viscoelastic equation with strong damping," Mathematical Methods in the Applied Sciences, vol. 24, no. 14, pp. 1043-1053, 2001.

[18] D. Ouchenane, K. Zennir, and M. Bayoud, "Global nonexistence of solutions for a system of nonlinear viscoelastic wave equation with degenerate damping and source terms," Ukrainian Mathematical Journal, vol. 65, no. 7, 2013.

[19] E. Pişkin and F. Ekinci, "Blow up of solutions for a coupled Kirchhoff-type equations with degenerate damping terms, applications and applied Mathematics," International Journal, vol. 14, no. 2, pp. 942-956, 2019.

[20] N. Mezouar and S. Boulaaras, "Global existence and decay of solutions of a singular nonlocal viscoelastic system with damping terms," Topological Methods in Nonlinear Analysis, vol. 1, 2020.

[21] F. Ekinci, E. Piskin, S. M. Boulaaras, and I. Mekawy, "Global existence and general decay of solutions for a quasilinear system with degenerate damping terms," Journal of function Spaces, vol. 2021, Article ID 4316238, 2021. 